\title{
Acute Renal Replacement Therapy in Intensive Care Units versus Outside Intensive Care Units: Are They Different?
}

This article was published in the following Dove Press journal: International Journal of Nephrology and Renovascular Disease

\author{
Daniela Ponce $\mathbb{1}^{1,2}$ \\ Welder Zamoner (D) ${ }^{2}$ \\ Vanessa Addad (iD) \\ Marci Maria Batistoco ${ }^{2}$ \\ André Balbi (DD ${ }^{1,2}$ \\ 'Internal Department, University State of \\ Sao Paulo- UNESP, Botucatu, Sao Paulo, \\ Brazil; ${ }^{2}$ Clinical Hospital of Botucatu \\ Medical School, Botucatu, Sao Paulo, \\ Brazil
}

\begin{abstract}
Introduction: Acute renal replacement therapy (RRT) is indicated when metabolic and fluid demands exceed total kidney capacity, and demand for kidney function is determined by non-renal comorbidities, severity of acute disease and solute and fluid burden; therefore, the criteria for commencing RRT and dialysis in intensive care units (ICUs) may be different to those outside ICUs. Objective: We investigated whether criteria for commencing acute RRT and dialysis outside ICU were different to those in ICU and whether these differences affected patient mortality in either setting.
\end{abstract}

Methods: We performed a retrospective observational study evaluating acute kidney injury (AKI), Kidney Disease Improving Global Outcome 3 (KDIGO3) in adult patients undergoing RRT "in and outside" ICU from 2012 to 2018, in a Brazilian teaching hospital.

Results: We evaluated 913 adults with AKI KDIGO3 undergoing RRT; 629 (68.9\%) outside ICU and 284 (31.1\%) in ICU. Infections were the main cause of hospitalisation (34.4\%). Septic and ischaemic AKI were the main aetiologies of AKI (50.8\% and 32.9\%, respectively), metabolic and fluid demand to capacity imbalance were the main indications for dialysis $(69.7 \%)$, and intermittent haemodialysis (IHD) was the primary dialysis method (59.2\%). The general mortality rate after 30 days was 59\%. There were no differences in gender, age and main diagnosis between groups. Both groups were different in acute tubular necrosis index specific scores (ATNISS), AKI aetiology, elderly population, indications for dialysis, dialysis methods and mortality rates. In ICU, patients older than 65 years old, with septic AKI were more prevalent (49.1 versus $41.4 \%$, and 55.1 versus $37.5 \%$, respectively), while ischaemic and nephrotoxic AKI were less frequent (24.3 versus 37 and 10.2 versus $16.3 \%$, respectively), and ATN-ISS was higher ( $0.74 \pm$ 0.31 versus $0.58 \pm 0.16$ ). Similarly, metabolic and fluid demand to capacity imbalance as an indication for acute RRT, prolonged intermittent haemodialysis (PIRRT) and continuous renal replacement therapy (CRRT) were more frequent, while peritoneal dialysis (PD) was less frequent ( 74.6 versus $69.7 \%$, 31.6 versus $22.4 \%$, and 5.3 versus $17.8 \%$, respectively), and mortality was higher (69 versus $54.7 \%$, respectively). Logistic regression revealed that age, septic AKI and being "in" ICU were factors associated with death.

Conclusion: The criteria for commencing RRT and dialysis in ICU were different to those outside ICU; however, they did not impact on patient outcomes.

Keywords: acute kidney injury, acute dialysis, mortality

\section{Introduction}

Acute renal replacement therapy (RRT) supports native renal function in correcting acid base, electrolyte disorders and fluid overload, and decreases the effects of acute kidney injury (AKI) on non-renal organs. ${ }^{1-3}$ Quality of life, social and cultural
Correspondence: Daniela Ponce Email daniela.ponce@unesp.br
International Journal of Nephrology and Renovascular Disease 2020:13 203-209 
issues, comorbid conditions, expected prognosis and logistics are also factors relevant to decision-making processes.

Macedo and Mehta initially described demand capacity balance in AKI; and recently it was recommended in a consensus ADQI meeting., ${ }^{4,5}$ The severity of acute illness, and solute and fluid burdens determine the demand. The demand capacity balance is dynamic and varies with the course of critical illness; therefore, it should be regularly evaluated. When kidney capacity reduces and fails to cope with demand, RRT initiation should be considered.

There is controversy regarding thresholds for whom and when RRT should be started for patients in ICU settings versus those outside settings should differ. The case-mix profiles of patients in ICU settings and nonICU settings are different and are largely characterised by differences in illness acuity and organ dysfunction burden. Currently, there are no data, either from clinical trials or observational studies, specifically comparing RRT provision between ICU and non-ICU settings.

Therefore, we investigated whether criteria for commencing acute RRT and dialysis outside ICU were different to those in ICU patients, and whether these differences affected patient mortality in either setting.

\section{Materials and Methods}

\section{Study Design and Setting}

Between 2012 and 2018, this retrospective cohort study was performed in AKI Kidney Disease Improving Global Outcome 3 (AKI KDIGO3) patients undergoing dialysis in wards and ICUs at the teaching hospital of the Botucatu School of Medicine (University of São Paulo State UNESP), Brazil. This is a major public hospital and treatment referral centre for approximately one-third of the population in São Paulo State. The study protocol was approved by the ethics committee of Botucatu School of Medicine in July 2012. Throughout the study, researchers adhered to patient data confidentiality and recommendations of the declaration of Helsinki. Free and informed consent was provided in writing by each patient or relatives before study commencement.

\section{Participants}

All AKI KDIGO3 hospitalised patients, treated by dialysis and consulted by nephrology teams were consecutively evaluated. The following patients were excluded; those with chronic kidney disease (CKD) stages III-V based on CKD-EPI, patients with previous kidney transplants or undergoing treatment with an RRT procedure, including haemodialysis or peritoneal dialysis (PD), and younger than 18 years. In addition, patients with glomerular missing data regarding a confirmed diagnosis were also excluded.

\section{Data Collection}

Study data were collected from patient medical files and filling out study checklists (see below). Those hospitalised AKI KDIGO3 patients, treated by dialysis were included. Their clinical profiles/data were extracted from archives.

Study checklists included demographic data (eg, age, sex), underlying illness, laboratory and ultrasonography findings, AKI aetiology (renal and post renal) and patient outcomes (ie, mortality). AKI diagnoses and causes were assessed by nephrologists in charge of patients at initial hospitalisation, and by referral to standard definitions. Renal AKI aetiology was classified as ischaemic or nephrotoxic acute tubular necrosis and septic, glomerular or interstitial tubular nephritis. Aetiology was also classified as mixed or uncertain.

AKI was defined according to KDIGO criteria. ${ }^{6}$ The criteria for nephrology consultation were based on physicians' individual criteria. After the nephrologist was consulted, it took $<12 \mathrm{~h}$ until arrival of the nephrologist to ICU or ward. Indications for dialysis included uraemia or azotaemia (BUN > $100 \mathrm{mg} / \mathrm{dL}$ ), fluid overload (after diuretic use), electrolyte imbalance (K.6.5 mEq/L after clinical treatment), acid-base disturbances $(\mathrm{pH} 7.1$ and $10 \mathrm{mEq} / \mathrm{L}$ bicarbonate after clinical treatment), and metabolic and fluid demand to capacity imbalance as defined by Macedo et al, and revised by Ostermann et al. ${ }^{4,5}$ The dialysis method was based on indications for dialysis, ie, haemodynamic stability and patient catabolism. The approach could be intermittent haemodialysis (IHD), prolonged intermittent RRT (PIRRT), continuous RRT (CRRT) and PD.

All AKI KDIGO3 dialysed patients were divided into two groups: "in ICU" and "outside ICU". The main goal was to evaluate and compare indications for acute dialysis. Clinical and laboratory characteristics, in-hospital mortality and kidney function recovery at discharge were also recorded.

\section{Statistical Analysis}

All analyses were performed using SPSS 13.0 statistical software (SPSS, Chicago, USA). To describe data, frequencies, percentages, means and standard deviations 
were used. For comparison of parametric variables between groups, the chi square statistical test and the Student's $t$-test were used. ANOVA, followed by Newman-Keuls tests, were used for multiple comparisons between groups. For nonparametric variables, Wilcoxon and Kruskal-Wallis tests, followed by Dunn's method, were used to compare two groups, and multiple groups, respectively. Variables with significant univariate associations such as age, prognostic scores, AKI aetiology, and "in ICU" and with the exit criteria set at $p<0.1$ were candidates for multivariable analysis. Multivariable logistic regression was performed using backward variable selection. Variables not selected by automated procedures were individually added back into models to evaluate for residual confounding and covariate.

Finally, collinearity amongst variables was tested, and if statistically significant interactions were present, one was excluded. Covariates included age and ATN-ISS, "in ICU” and ATN-ISS, "in ICU” and dialysis methods (prolonged HD and no PD), "in ICU” and dialysis indications (metabolic and fluid demand to capacity imbalance). We considered $\mathrm{p}<0.05$ as statistically significant.

\section{Results}

During the seven-year study period, 7976 AKI patients were evaluated by the nephrology team. After excluding patients with CKD stages III-V, kidney transplants, those with incomplete data and AKI KDIGO 1, 2 and 3 not treated by dialysis, 913 (16.8\%) patients were included, therefore these patients were treated by acute RRT.

The main characteristics of the general AKI KDIGO3 population, treated by dialysis, are shown (Table 1). The mean age was $61.45 \pm 16.75$ years; $62.3 \%$ of patients were male and $76.4 \%$ were Caucasian. The mean ATN-ISS was $0.71 \pm 0.28$, and $31.1 \%$ of patients were "in" ICU. Infections were the main cause of hospitalisation, septic and ischaemic AKI were the main AKI aetiologies, metabolic and fluid demand to capacity imbalance were the main indications for dialysis, and IHD was the main dialysis method. The mortality rate after 30 days was $59 \%$.

Table I Clinical Data of Acute Kidney Injury Patient Followed by Nephrology Team According to Being IN vs OUTSIDE ICU

\begin{tabular}{|c|c|c|c|c|}
\hline & General $(N=913)$ & IN ICU $(N=284)$ & OUTSIDE ICU (N = 629) & $\mathbf{P}$ \\
\hline Age (years) & $61.65 \pm 16.26$ & $61.13 \pm 15.23$ & $61.45 \pm 16.75$ & 0.56 \\
\hline Age $>65$ years old $(\%)$ & $401(43.9)$ & $|4|(49.6)$ & $260(4 I .4)$ & 0.02 \\
\hline Male sex (\%) & $569(62.3)$ & $180(63.3)$ & $389(62.1)$ & 0.57 \\
\hline Caucasian patients (\%) & $698(76.4)$ & $218(76.8)$ & 501 (79.9) & 0.51 \\
\hline Main diagnosis (\%) & & & & $>0.05$ \\
\hline Cardiovascular disease & $193(21.1)$ & $56(19.7)$ & $137(2 \mid .8)$ & \\
\hline Infectious & $314(34.4)$ & $100(35.2)$ & $214(34.1)$ & \\
\hline Pos surgery & $26(2.8)$ & $15(5.3)$ & II (I.7) & \\
\hline Neurologic disorders & $28(3.1)$ & $10(3.5)$ & $18(2.9)$ & \\
\hline Trauma & $72(7.9)$ & $33(11.6)$ & $39(6.2)$ & \\
\hline Liver failure & $4 \mid(4.5)$ & II (3.9) & $30(4.8)$ & \\
\hline Malignancy & $82(8.9)$ & $25(8.8)$ & $57(9.1)$ & \\
\hline Shock & $51(5.6)$ & $13(4.6)$ & $38(6.1)$ & \\
\hline Others & $66(7.2)$ & $14(4.9)$ & $52(8.2)$ & \\
\hline Uncertain & $40(4.4)$ & $6(2.1)$ & $34(5.4)$ & \\
\hline AKI etiology (\%) & & & & $<0.05$ \\
\hline \multicolumn{5}{|l|}{ Renal } \\
\hline Sepsis (\%) & $393(50.8)$ & $158(55.1)^{\mathrm{a}}$ & $235(37.5)^{\mathrm{b}}$ & \\
\hline Ischemic ATN & $301(32.9)$ & $69(24.3)^{\mathrm{a}}$ & $232(37)^{\mathrm{b}}$ & \\
\hline Nephrotoxic ATN & $131(14.4)$ & $29(10.2)^{a}$ & $102(16.3)^{b}$ & \\
\hline Others** & $14(1.5)$ & $2(0.7)$ & $12(1.9)$ & \\
\hline Pos renal & $4(0.4)$ & $\mathrm{I}(0.3)$ & $3(0.4)$ & \\
\hline ATN-ISS & $0.7 I \pm 0.28$ & $074 . \pm 0.31$ & $0.58 \pm 0.16$ & $<0.001$ \\
\hline Mortality after 30 days (\%) & $539(59)$ & $196(69)$ & $343(54.7)$ & $<0.001$ \\
\hline
\end{tabular}

Notes: **Others: interstitial tubular nephritis and glomerular AKI, uncertain.

Abbreviations: AKI, acute kidney injury; ATN-ISS, acute tubular necrosis index score specific; ICU, intensive care unit. 
Table 2 Indications for Dialysis in Acute Kidney Injury Patient Followed by Nephrology Team According to Being IN vs OUTSIDE ICU

\begin{tabular}{|c|c|c|c|c|}
\hline & General $(\mathbf{N}=913)$ & IN ICU $(N=284)$ & OUTSIDE ICU (N = 629) & $\mathbf{P}$ \\
\hline Dialysis indication (\%) & & & & $<0.05$ \\
\hline Uremia & $87(9.5)$ & $17(6)^{\mathrm{a}}$ & $70(11.2)^{b}$ & \\
\hline Refractory Hyperkalemia & $47(5.1)$ & $13(4.6)$ & $34(5.4)$ & \\
\hline Hypervolemia & $97(10.6)$ & $32(11.6)$ & $66(10.4)$ & \\
\hline Metabolic and fluid demand to capacity imbalance & $637(69.7)$ & $212(74.6)^{\mathrm{a}}$ & $425(67.5)^{\mathrm{b}}$ & \\
\hline Refractory acidosis & $45(1.8)$ & $12(4.2)$ & $33(5.3)$ & \\
\hline Dialysis Methods (\%) & & & & $<0.05$ \\
\hline Intermittent Hemodialysis & $540(59.2)$ & $166(58.5)$ & $374(59.5)$ & \\
\hline Prolonged Hemodialysis & $231(25.3)$ & $90(31.6)^{a}$ & $|4|(22.4)^{b}$ & \\
\hline Continuous renal replacement therapy & $15(1.6)$ & $13(4.5)^{\mathrm{a}}$ & $2(0.3)^{\mathrm{b}}$ & \\
\hline Peritoneal dialysis & $127(13.9)$ & $15(5.3)^{\mathrm{a}}$ & $112(17.8)^{b}$ & \\
\hline
\end{tabular}

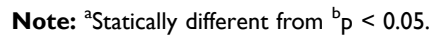

Abbreviation: ICU, intensive care unit.

The characteristics of AKI KDIGO3 patients treated by dialysis "in" ICU versus those "outside" ICU are shown (Table 1). There were no differences between both groups in terms of gender, age and main diagnosis. Both groups were different for ATN-ISS, AKI aetiology, elderly population, indications for dialysis, dialysis methods and mortality rates. "In" ICU patients $>65$ years and with septic AKI were the most prevalent, while ischaemic and nephrotoxic AKI were less frequent, and ATN-ISS was higher. Metabolic and fluid demand to capacity imbalance, prolonged haemodialysis and CRRT were more frequent in "in" ICU patients, while PD was less frequent and mortality was higher (Table 2). Using logistic regression, age, septic AKI, and being "in" ICU were associated with mortality (Table 3).

\section{Discussion}

Using a large, nationally representative database of Brazilian AKI KDIGO3 patients undergoing dialysis "in and outside ICU" between 2012 and 2018, we observed that for "in" ICU, patients $>65$ years old, with septic AKI were more frequent than "outside" ICU, while ischaemic

Table 3 Distribution Hazard Ratio of Covariates for Mortality

\begin{tabular}{|l|l|l|}
\hline Variables & HR (95\% CI) & $\mathbf{p}$ \\
\hline Age $>65$ years & $1.08(1.02-1.75)$ & 0.04 \\
In ICU & $2.12(1.73-3.66)$ & 0.003 \\
Septic AKI & $1.18(1.04-1.97)$ & 0.04 \\
Uremia & $0.97(0.91-2.76)$ & 0.31 \\
CRRT & $1.54(0.97-2.67)$ & 0.34 \\
\hline
\end{tabular}

Abbreviations: AKI, acute kidney injury; CRRT, continuous renal replacement therapy; HR, hazard ratio; ICU, intensive care unit. and nephrotoxic AKI were less frequent. In relation to indications for dialysis, metabolic and fluid demand to capacity imbalance was more frequent, while uraemia was less frequent, when compared to "outside" ICU patients. PIRRT and CRRT were more frequent, while (PD) was less used than "outside" ICU patients. Mortality rates were higher for "in" ICU patients.

After adjusting for confusing variables, we generated three independent mortality predictors: aged $\geq 65$, ICU admission and septic AKI. Indications for dialysis and dialysis methods were not associated with death.

Several studies have showed that AKI patients in ICU are associated with higher mortality. ${ }^{7-10}$ Seeing that ICU patients are in severe condition due to increased sepsisassociated AKI, increased aged and ATN-ISS, being more affected by multiorgan failure and having pre-existing chronic comorbidities, these unfavourable outcomes can be justified.

Considering that acute RRT should be considered when metabolic and fluid demands exceed renal capacity and that demand for kidney function is determined by nonrenal comorbidities, the severity of acute disease and solute and fluid burden, the criteria for starting RRT in ICU vs outside ICU could be different and affect patient survival. There is a lack of evidence on the optimal timing of dialysis for "in" versus "outside" ICU. ${ }^{4-6,11}$

Several factors should be considered whether to initiate RRT for AKI. It is accepted that dialysis should be rapidly initiated in patients with life-threatening indications, such as severe acidosis and hyperkalaemia. However, apart from these indications, KDIGO clinical practice guidelines recommend considering the clinical context of the patient, ${ }^{6}$ eg fluid 
overload, illness severity, organ dysfunction, urine output and solute burden, and the capacity for renal function recovery. ${ }^{7,11}$

Macedo and Mehta described demand capacity balance for AKI, and later it was recommended by consensus at an ADQI meeting. ${ }^{4,5}$ The severity of acute illness and solute and fluid burden determine demand. The demand capacity balance is dynamic and varies with the course of critical illness. When kidney capacity reduces and fails to cope with these demands, RRT should be initiated. ${ }^{5}$

In this study, the criteria for commencing acute RRT were different between "in" and "outside" ICU patients. The demand capacity imbalance was more frequent in "in" ICU patients, while uraemia was less frequent, however they were not associated with death. Recently, five randomised controlled trials (RCTs) compared early RRT initiation versus late, ${ }^{12-16}$ with controversial results. Based on existing clinical evidence and context, the decision to start acute RRT should be individualised, and not based solely on renal function or AKI stage. ${ }^{11}$ Quality of life, social and cultural issues, logistics, comorbid conditions and expected prognoses are also relevant factors in decisionmaking processes. In low-income countries, physicians may have ethical dilemmas in initiating RRT in patients with poor prognoses; therefore, a shared decision process with the patient and family should be undertaken. ${ }^{11}$

Clinical decision systems should be implemented to help health-care workers decide when and how to start acute RRT. Such systems should investigate thresholds for demand-capacity imbalance (magnitude and duration) for which acute RRT commencement causes different clinical outcomes.

There is also a lack of evidence on acute RRT method choices. We believe such choices are based on local availability, team experience with specific treatments, and patient clinical status. ${ }^{11}$ RRT modalities for AKI include IHD, PIRRT, CRRT and PD. From the literature, at least 10 RCTs, and three systematic reviews and meta-analyses have compared CRRT and IHD. ${ }^{8,17-28}$ All observed no significant differences in mortality or recovery of renal function between patients treated with intermittent or continuous modalities. ${ }^{8,17-28}$ Based on these observations, KDIGO clinical practice guidelines for AKI have suggested continuous and intermittent RRT modalities as complementary, except for patients with acute brain injury or haemodynamic instability, for whom CRRT or PD are the first options. ${ }^{20}$ Hybrid treatments are used for haemodynamically unstable patients, in situations where CRRT is not available, ${ }^{8,26-31}$ however comparative efficacy data are limited. ${ }^{29-34}$
A systematic review and meta-analysis, including 17 studies from 2000 to 2014, focused on the impact of PIRRT and CRRT on mortality and kidney recovery. ${ }^{32}$ When only RCTs were included in the analysis, no differences in mortality were observed between both modalities, however, when data from observational studies were analysed, PIRRT was associated with lower mortality. There were no significant differences in the recovery of kidney function, fluid removal, days in ICU, and biochemical efficacy in both RCTs and observational studies. This meta-analysis concluded that PIRRT was associated with similar outcomes to CRRT. ${ }^{32}$

PD experience in AKI is limited, except in paediatric settings and regions with limited resources. ${ }^{35-43} \mathrm{PD}$ is attractive for a selective group of patients because it is a simple procedure, and it provides RRT without machinery and electricity. Technical advances (ie, flexible catheters, automatic cycling and high and continuous volumes) have made $\mathrm{PD}$ an acceptable alternative to $\mathrm{HD}$. The International Society for Peritoneal Dialysis (ISPD) recommends that $\mathrm{PD}$ is a suitable modality for $\mathrm{AKI}$ patients, especially in low-income countries. ${ }^{44}$ Recent studies have confirmed decreases in mortality and complications related to $\mathrm{PD}$ in countries where acute $\mathrm{PD}$ is regularly performed. ${ }^{37-45}$ IHD is the preferred treatment for the immediate removal of small solutes, such as severe hyperkalaemia, poisoning and tumour lysis syndrome. ${ }^{20}$

In this study, we observed differences in dialysis methods between patients "in" and "outside" ICU. PD was indicated more frequently "outside" ICU, whilst PIRRT and CRRT were more frequently indicated "in" ICU. However, these dialysis methods were not associated with mortality. Based on this evidence, RRT modality should be based on modality availability, clinical experience, cost of therapy and patient clinical status (haemodynamic stability, need for fluid removal, catabolic state, lifethreatening complications or acute brain injury).

Our study had several strengths, most notably its large sample size and duration. Its major limitations were the absence of detailed laboratory and clinical data, and its mono-centric status. Although criteria for commencing acute RRT and dialysis are different between AKI patients "in" and "outside" ICU, they did not affect short patients' survival. Admission to ICU, age and septic AKI were risk factors associated with death.

Finally, acute RRT should be rapidly initiated both "in and outside" ICU, when life-threatening changes in electrolytes, fluids, and acid-base balances are unresponsive to medical therapies. Rapid RRT should be considered when metabolic 
and fluid demands exceed total renal capacity. Future studies should therefore evaluate other factors that impact on decision-making for RRT "in and outside" ICU, such as quality of life, expected prognosis, social and cultural issues, comorbid conditions, severity of acute illness and logistics.

\section{Ethics Statement}

We confirm all methods were performed in accordance with relevant guidelines and regulations from Botucatu Medical School. The research project was approved by the Ethics Committee of the Botucatu Medical School in June 2012 (IRB approval number 0641/012). Free and informed consent was given in writing by each patient or relatives before the commencement of study procedures.

\section{Acknowledgment}

We thank all nurse teams from the dialysis unit at UNESP, Botucatu, Brazil.

The partial results of this manuscript were presented in The Twenty-Fifth Annual International Conference on Advances in Critical Care Nephrology February 24-27, 2020 AKI \& CRRT 2020 Conference San Diego, California as oral poster presentation titled: Should The Criteria For Starting Acute RRT in ICU vs Outside ICU Be Different?

\section{Author Contributions}

All authors made substantial contributions to conception and design, acquisition of data, or analysis and interpretation of data; took part in drafting the article or revising it critically for important intellectual content; gave final approval of the version to be published; and agree to be accountable for all aspects of the work.

\section{Funding}

Coordenação de Aperfeiçoamento de Pessoal de Nível Superior (CAPES) supported this study.

\section{Disclosure}

The authors declare no competing financial and nonfinancial interests in this work.

\section{References}

1. Ostermann M, Chang RW. Correlation between parameters at initiation of renal replacement therapy and outcome in patients with acute kidney injury. Crit Care. 2009;13:R175. doi:10.1186/cc8154

2. Macedo E, Mehta RL. Continuous dialysis therapies: core curriculum. Am J Kidney Dis. 2016;68(4):645-657. doi:10.1053/j.ajkd.2016.03. 427
3. Mehta RL. Challenges and pitfalls when implementing renal replacement therapy in the ICU. Crit Care. 2015;19(3):S9. doi:10.1186/cc14727

4. Macedo E, Mehta RL. When should renal replacement therapy be initiated for acute kidney injury? Semin Dial. 2011;24:132-137. doi:10.1111/j.1525-139X.2011.00838.x

5. Ostermann M, Joannidis M, Pani A, et al. 17th acute disease quality initiative (ADQI) consensus group patient selection and timing of continuous renal replacement therapy. Blood Purif. 2016;42 (3):224-237. doi:10.1159/000448506

6. Kidney disease: improving global outcomes (KDIGO) acute kidney injury work group. KDIGO clinical practice guidelines for acute kidney injury. Kidney Int Suppl. 2012;2:1-141.

7. Kitchlu A, Adhikari N, Burns KE, et al. Outcomes of sustained low efficiency dialysis versus continuous renal replacement therapy in critically ill adults with acute kidney injury: a cohort study. $B M C$ Nephrol. 2015;16:127. doi:10.1186/s12882-015-0123-4

8. Ponce D, Abrão JM, Albino BB, et al. Extended daily dialysis in acute kidney injury patients: metabolic and fluid control and risk factors for death. PLoS One. 2013;8:e81697. doi:10.1371/journal. pone. 0081697

9. Costa e Silva VT, Liãno F, Muriel A, et al. Nephrology referral and outcomes in critically Ill acute kidney injury patients. PLoS One. 2013;8(8):e70482. doi:10.1371/journal.pone.0070482

10. Lima EQ, Zanetta DMT, Castro I, Yu L. Mortality risk factors and validation of severity scoring systems in critically ill patients with acute renal failure. Ren Fail. 2005;27:547-556. doi:10.1080/08860220500198771

11. Annigeri RA, Ostermann M, Tolwani A, et al. The acute dialysis quality initiative (ADQI) consensus group renal support for acute kidney injury in the developing world. Kidney Int Rep. 2017;2:559-578. doi:10.1016/j.ekir.2017.04.006

12. Karvellas CJ, Farhat MR, Sajjad I, et al. A comparison of early versus late initiation of renal replacement therapy in critically ill patients with acute kidney injury: a systematic review and meta-analysis. Crit Care. 2011;15:R72. doi:10.1186/cc10061

13. Wierstra BT, Kadri S, Alomar S, et al. The impact of 'early' versus 'late' initiation of renal replacement therapy in critical care patients with acute kidney injury: a systematic review and evidence synthesis. Crit Care. 2016;20:122. doi:10.1186/s13054-016-1291-8

14. Wald R, Adhikari NK, Smith OM, et al. Comparison of standard and accelerated initiation of renal replacement therapy in acute kidney injury. Kidney Int. 2015;88:897-904. doi:10.1038/ki.2015.184

15. Zarbock A, Kellum JA, Schmidt C, et al. Effect of early vs. delayed initiation of renal replacement therapy on mortality in critically ill patients with acute kidney injury: the ELAIN randomised clinical trial. JAMA. 2016;315:2190-2199. doi:10.1001/jama.2016.5828

16. Gaudry S, Verney C, Hajage D, et al. Hypothesis: early renal replacement therapy increases mortality in critically ill patients with acute on chronic renal failure. A post hoc analysis of the AKIKI trial. Intensive Care Med. 2018;44:1360-1361. doi:10.1007/s00134-018-5210-0

17. Vaara S, Reinikainen M, Wald R, et al. Timing of RRT based on the presence of conventional indications. Clin J Am Soc Nephrol. 2014;9:1577-1585. doi:10.2215/CJN.12691213

18. Gasparovic V, Filipovic-Grcic I, Merkler M, et al. Continuous renal replacement therapy (CRRT) or intermittent haemodialysis (IHD) what is the procedure of choice in critically ill patients? Ren Fail. 2003;25:855-862. doi:10.1081/JDI-120024300

19. Vinsonneau C, Camus C, Combes A, et al. Continuous venovenous haemodiafiltration versus intermittent haemodialysis for acute renal failure in patients with multipleorgan dysfunction syndrome: a multicentre randomised trial. Lancet. 2006;368:379-385. doi:10.1016/S0140-6736(06)69111-3

20. Lins RL, Elseviers MM, Van der Niepen P, et al. Intermittent versus continuous renal replacement therapy for acute kidney injury patients admitted to the intensive care unit: results of a randomised clinical trial. Nephrol Dial Transplant. 2009;24:512-518. doi:10.1093/ndt/ gfn560 
21. Kielstein JT, Kretschmer U, Ernst T, et al. Efficacy and cardiovascular tolerability of extended dialysis in critically ill patients: a randomised controlled study. Am J Kidney Dis. 2004;43:342-349. doi:10.1053/j.ajkd.2003.10.021

22. Mehta RL, McDonald B, Gabbai FB, et al. A randomised clinical trial of continuous versus intermittent dialysis for acute renal failure. Kidney Int. 2001;60:1154-1163. doi:10.1046/j.1523-1755.2001.06 00031154.x

23. Bagshaw SM, Berthiaume LR, Delaney A, et al. Continuous versus intermittent renal replacement therapy for critically ill patients with acute kidney injury: a meta-analysis. Crit Care Med. 2008;36:610-617. doi:10.1097/01.CCM.0B013E3181611F552

24. Pannu N, Klarenbach S, Wiebe N, et al. Renal replacement therapy in patients with acute renal failure: a systematic review. JAMA. 2008;299:793-805. doi:10.1001/jama.299.7.793

25. Rabindranath K, Adams J, Macleod AM, et al. Intermittent versus continuous renal replacement therapy for acute renal failure in adults. Cochrane Database Syst Rev. 2007;CD003773.

26. Kumar VA, Craig M, Depner TA, et al. Extended daily dialysis: a new approach to renal replacement for acute renal failure in the intensive care unit. Am J Kidney Dis. 2000;36:294-300. doi:10.1053/ ajkd.2000.8973

27. Albino BB, Balbi AL, Abrao JM, et al. Dialysis complications in acute kidney injury patients treated with prolonged intermittent renal replacement therapy sessions lasting 10 versus 6 hours: results of a randomised clinical trial. Artif Organs. 2015;39:423-431. doi:10.1111/aor. 12408

28. Fieghen HE, Friedrich JO, Burns KE, et al. The haemodynamic tolerability and feasibility of sustained low efficiency dialysis in the management of critically ill patients with acute kidney injury. $B M C$ Nephrol. 2010;11:32. doi:10.1186/1471-2369-11-32

29. Marshall MR, Ma T, Galler D, et al. Sustained low-efficiency daily diafiltration (SLEDD-f) for critically ill patients requiring renal replacement therapy: towards an adequate therapy. Nephrol Dial Transplant. 2004;19:877-884. doi:10.1093/ndt/gfg625

30. Holt BG, White JJ, Kuthiala A, et al. Sustained low-efficiency daily dialysis with haemofiltration for acute kidney injury in the presence of sepsis. Clin Nephrol. 2008;69:40-46. doi:10.5414/CNP69040

31. Kumar VA, Yeun JY, Depner TA, et al. Extended daily dialysis vs. continuous haemodialysis for ICU patients with acute renal failure: a two-year single centre report. Int J Artif Organs. 2004;27:371-379. doi:10.1177/039139880402700505

32. Zhang L, Yang J, Eastwood GM, et al. Extended daily dialysis versus continuous renal replacement therapy for acute kidney injury: a meta-analysis. Am J Kidney Dis. 2015;66:322-330. doi:10.1053/j. ajkd.2015.02.328
33. Bell M, Granath F, Schon S, et al. Continuous renal replacement therapy is associated with less chronic renal failure than intermittent haemodialysis after acute renal failure. Intensive Care Med. 2007;33:773-780. doi:10.1007/s00134-007-0590-6

34. Farese S, Jakob SM, Kalicki R, et al. Treatment of acute renal failure in the intensive care unit: lower costs by intermittent dialysis than continuous venovenous haemodiafiltration. Artif Organs. 2009;33: 634-640. doi:10.1111/j.1525-1594.2009.00794.x

35. Ponce D, Berbel MN, Regina de Goes C, et al. High volume peritoneal dialysis in acute kidney injury: indications and limitations. Clin J Am Soc Nephrol. 2012;7:887-894. doi:10.2215/CJN.11131111

36. Ponce D, Buffarah MB, Goes C, et al. Peritoneal dialysis in acute kidney injury: trends in the outcome across time periods. PLoS One. 2015;10:e0126436. doi:10.1371/journal.pone.0126436

37. Remuzzi G, Horton R. Acute renal failure: an unacceptable death sentence globally. Lancet. 2013;382:2041-2042. doi:10.1016/S01406736(13)62193-5

38. Perico N, Remuzzi G. Acute kidney injury in low-income and middle-income countries: no longer a death sentence. Lancet Glob Health. 2016;4:e216-e217. doi:10.1016/S2214-109X(16)00065-6

39. Callegari JG, Kilonzo KG, Yeates KE, et al. Peritoneal dialysis for acute kidney injury in sub-Saharan Africa: challenges faced and lessons learned at Kilimanjaro Christian Medical Centre. Kidney Int. 2012;81:331-333. doi:10.1038/ki.2011.408

40. Abdelraheem M, Ali ET, Osman R, et al. Outcome of acute kidney injury in Sudanese children - an experience from a sub-Saharan African unit. Perit Dial Int. 2014;34:526-533. doi:10.3747/ pdi.2013.00082

41. Finkelstein FO, Smoyer WE, Carter M, et al. Peritoneal dialysis, acute kidney injury, and the saving young lives program. Perit Dial Int. 2014;34:478-480. doi:10.3747/pdi.2014.00041

42. Kilonzo KG, Ghosh S, Temu SA, et al. Outcome of acute peritoneal dialysis in northern Tanzania. Perit Dial Int. 2012;32:261-266. doi: $10.3747 /$ pdi.2012.00083

43. Cullis B, Abdelraheem M, Abrahams G, et al. ISPD guidelines/ recommendations peritoneal dialysis for acute kidney injury. Perit Dial Int. 2014;34:494-517. doi:10.3747/pdi.2013.00222

44. Ronco C, Bellomo R, Brendolan A, et al. Brain density changes during renal replacement in critically ill patients with acute renal failure. Continuous haemofiltration versus intermittent haemodialysis. J Nephrol. 1999;12:173-178.

45. Davenport A, Will EJ, Davison AM. Effect of renal replacement therapy on patients with combined acute renal and fulminant hepatic failure. Kidney Int Suppl. 1993;41:S245-S251.
The International Journal of Nephrology and Renovascular Disease is an international, peer-reviewed open-access journal focusing on the pathophysiology of the kidney and vascular supply. Epidemiology, screening, diagnosis, and treatment interventions are covered as well as basic science, biochemical and immunological studies. The manuscript management system is completely online and includes a very quick and fair peer-review system, which is all easy to use. Visit http://www.dovepress.com/testimonials.php to read real quotes from published authors 